\title{
Comparison of Outcomes of Resident-performed Ahmed Valve Implantation vs Trabeculectomy
}

\author{
${ }^{1}$ Robert A Sharpe, ${ }^{2}$ Leah L Kammerdiener, ${ }^{3}$ Kendall W Wannamaker, ${ }^{4}$ Jie Fan, ${ }^{5}$ Elizabeth D Sharpe
}

\begin{abstract}
Aims: To compare outcomes of resident-performed Ahmed valve surgery vs trabeculectomy in a Veteran Affairs medical facility.

Materials and methods: A retrospective cohort of 103 eyes in 91 patients receiving Ahmed valve (valve) or trabeculectomy (trab) performed at a Veterans Administration Medical Center by residents in their third year of training. The primary outcomes included intraocular pressure (IOP), treatment failure, and complications over 1 year.
\end{abstract}

Results: Of 103 eyes, 44 received valve and 59 received trab. Primary open-angle glaucoma was primary diagnosis more often in trab, while neovascular glaucoma predominated in the valve group $(p<0.001)$. Preoperative mean IOP was $35.1 \pm 11.8$ and $24.5 \pm 7.1 \mathrm{~mm} \mathrm{Hg}$ for valve and trabeculectomy respectively $(p<0.001)$, but at 1 year the IOP difference between groups was not statistically significant $(p=0.064)$. Overall, $11(25.0 \%)$ and $11(18.6 \%)$ eyes met any criteria for failure for valve and trab respectively. At 1 year, $22.5 \%$ of valves had IOP $>21 \mathrm{~mm} \mathrm{Hg}$ vs only $4.3 \%$ of trab $(p=0.02)$. Complications were infrequent. There were no intraoperative complications for valve, whereas five for trab. Most common immediate complication for valve was hyphema. Both groups had low rates of choroidal effusions and reoperation.

Conclusion: Ahmed valve implantation and trabeculectomy produce significant reductions in IOP when performed by residents-in-training. Valves tend to be used more frequently in patients with secondary glaucoma. Although complication profiles differ between procedures, both are safe and well tolerated when performed by resident physicians.

Clinical significance: This study provides support for evidence-based patient counseling that supervised, residentperformed Ahmed valve implantation and trabeculectomy are indeed safe and effective.

Keywords: Glaucoma, Resident, Trabeculectomy, Training, Valve.

How to cite this article: Sharpe RA, Kammerdiener LL, Wannamaker KW, Fan J, Sharpe ED. Comparison of Outcomes of Resident-performed Ahmed Valve Implantation vs Trabeculectomy. J Curr Glaucoma Pract 2016;10(2):60-67.

Source of support: Nil

Conflict of interest: Authors report no conflicts of interest and received no financial support from any private or government

\footnotetext{
${ }^{1,3}$ Resident, ${ }^{2}$ Fellow, ${ }^{4}$ Research Instructor, ${ }^{5}$ Professor

${ }^{1-4}$ Department of Ophthalmology, Medical University of South Carolina, Charleston, South Carolina, USA

${ }^{5}$ Department of Ophthalmology, Ralph H. Johnson Veterans Affairs Medical Center, Charleston, South Carolina, USA

Corresponding Author: Robert ASharpe, Resident, Department of Ophthalmology, Medical University of South Carolina Charleston, South Carolina, USA, Phone: +8437922020, e-mail: sharpera@musc.edu
}

funding source for this study. This study was approved by the Medical University of South Carolina Institutional Review Board and the Veterans Affairs Office of Research and Development.

\section{INTRODUCTION}

Glaucoma is a progressive optic neuropathy, i.e., a leading cause of irreversible blindness worldwide. ${ }^{1}$ The mainstay of treatment centers on reducing intraocular pressure (IOP). Surgical procedures are commonly employed in the management of glaucoma when medical and laser treatments are inadequate or not tolerated. ${ }^{2}$

Trabeculectomy, first described in 1968, remains the gold standard and most commonly performed incisional glaucoma surgery. ${ }^{3-5}$ Trabeculectomy is typically performed with antifibrotic agents, most commonly mitomycin C, to improve the success rates of bleb function. ${ }^{6}$ Despite its widespread acceptance, trabeculectomy with antifibrotic agents is a technically demanding procedure and requires careful follow-up to produce optimal aqueous outflow without overfiltration.

The Ahmed valve (New World Medical, Rancho Cucamonga, California) is a tube shunt that has traditionally been reserved for select patients with an increased risk of trabeculectomy failure or a high risk of secondary glaucoma. ${ }^{7,8}$ More recently, tube shunts have grown in popularity as the first-line surgery for glaucoma. 3,4,6,9 The advantage of the Ahmed valve, like all tube shunts, lies in its relatively straightforward implantation process and has been shown to require fewer postoperative interventions than the nonvalved Baerveldt at 1 year. ${ }^{10}$

During their 3 years of training, ophthalmology residents are required to perform various surgical treatments for glaucoma. The American College of Graduate Medical Education requires residents to log at least five filtering or shunt surgeries as primary surgeon before graduation. ${ }^{11}$ Eighty-four percent of residents receive surgical instruction by fellowship-trained glaucoma specialist and act as primary surgeon for a tube shunt by their third year. ${ }^{12}$ On average, residents perform 3.6 tube shunts and 8.6 trabeculectomies during training. ${ }^{12}$ Adequate exposure is essential during residency because comprehensive ophthalmologists, who generally do not complete fellowships, commonly perform these procedures when medically necessary. ${ }^{13}$

This study aims to investigate Ahmed valve implantation outcomes compared with trabeculectomy when 
performed by residents-in-training. Connor et $\mathrm{al}^{14}$ published a similar study in 2010, but compared trabeculectomy with Baerveldt devices. To our knowledge, no other study has investigated IOP outcomes and complication rates of these two glaucoma surgeries performed by residents to date.

\section{MATERIALS AND METHODS}

A retrospective case review was performed at the Ralph H Johnson Veterans Affairs Medical Center (VAMC) in Charleston, SC. The study was approved by the Medical University of South Carolina Institutional Review Board and the VAMC Office of Research and Development. All cases were performed by residents in their third year of ophthalmology training. All stages of this study were conducted in accordance with the principles set forth by the Declaration of Helsinki.

Patients who underwent glaucoma surgery from 2005 to 2012 were identified via surgery schedules. All included surgeries were performed under the supervision of a single, fellowship-trained glaucoma surgeon. From that list, only primary Ahmed valve implantation and trabeculectomy cases were included. Preoperative clinic visits within 6 months of the surgical dates were used for baseline parameters. Patients were then followed as a retrospective cohort for 1 year postoperatively.

Patients with prior incisional glaucoma surgery were excluded from this study. Additional exclusion criteria consisted of loss to follow-up before 1 month postoperatively, revision procedures, and combination procedures including cyclocryotherapy, cyclophotocoagulation, limbal relaxing incisions, intravitreal injections, and vitrectomy. In addition, combination Ahmed valve-phacoemulsification and phacoemulsificationtrabeculectomy were excluded.

Demographic data including age, sex, race, history of diabetes, and history of hypertension were collected. Ocular history was also obtained, including glaucoma diagnosis, prior nonglaucoma ophthalmic surgery, and prior glaucoma laser treatment. Intraocular pressure, number of ocular antihypertensive medications, bestcorrected visual acuity (BVCA), and cup-to-disk ratio (C/D) were evaluated preoperatively and at 1 year postoperatively. Preoperative IOP was calculated by taking the mean IOP from 2 visits within 6 months of surgery. Intraocular pressure was also measured at 1 day, 1 week, 1, 3, 6 months, and 1 year. Intra- and postoperative complications were recorded over 1 year. Complications over 1 year were recorded for each type of surgery. Time course of complications was defined as immediate, early, or late for those occurring at $<1$ week, 1 week to 3 months, and 3 to 6 months respectively. Overfiltration (i.e., hypotony) was defined as were defined as IOP $<5 \mathrm{~mm} \mathrm{Hg}$.
After surgery, patients returned at standard intervals for routine postoperative care unless a complication arose that necessitated closer follow-up. Patients in all groups received topical steroids (prednisolone acetate $1 \%$ ) and antibiotics (most commonly moxifloxacin $0.5 \%$ ) postoperatively. At each follow-up appointment, BCVA was obtained by refraction or pinhole, IOP was measured with Goldmann applanation tonometry, and slit-lamp biomicroscopy was performed. Indirect ophthalmoscopy was performed to evaluate for choroidal effusion when the IOP was $<5 \mathrm{~mm} \mathrm{Hg}$. Suture lysis was performed as needed with Argon laser between 1 and 3 weeks after trabeculectomy to increase outflow using clinical judgment based on IOP and health of optic nerve.

Criteria for failure were modeled after the Tube vs Trabeculectomy study. ${ }^{15}$ Treatment failure included IOP $>21 \mathrm{~mm} \mathrm{Hg}$, IOP not reduced by $20 \%$ below baseline on two consecutive follow-up visits after 3 months postoperatively, or IOP $\leq 5 \mathrm{~mm} \mathrm{Hg}$ on two consecutive follow-up visits after 3 months. If data from two consecutive visits after 3 months were not available, then the eye was excluded from treatment failure analysis.

Ahmed valves were implanted superotemporally using standard surgical technique. A peribulbar block was administered using 5 cc of a $50 \%$ mixture of $2 \%$ lidocaine and $0.75 \%$ bupivacaine. A $6-0$ plain gut bridle suture was used to rotate the eye inferonasally. Conjunctiva and Tenon's capsule were then bluntly dissected and undermined anteriorly and posteriorly. The Ahmed valve was tested for functionality and then placed into the wound and positioned posteriorly. It was sutured in place approximately $7 \mathrm{~mm}$ posterior to the limbus, and the tube was cut and beveled to fit 2 to $3 \mathrm{~mm}$ into the anterior chamber (AC). A 23-gauge needle was used to create a tract starting $0.5 \mathrm{~mm}$ from the limbus into the $\mathrm{AC}$, running parallel to the plane of the iris, and Healon was injected into the AC. The tube was then placed in the tract and positioned in the AC. A 10-0 nylon suture was used to secure the tube to the sclera. A Tutoplast graft patch (IOP Ophthalmics, Costa Mesa, California) was then sutured with 10-0 nylon over the valve and tube. Tenon's capsule and conjunctiva were reapproximated and closed, and neomycin/polymyxin $\mathrm{B} /$ dexamethasone ophthalmic ointment was applied at the end of the case.

A superotemporal or superonasal approach was employed for all trabeculectomies. Peribulbar block with 5 cc of a $50 \%$ mixture of $1 \%$ lidocaine and $0.75 \%$ bupivicaine was performed. The eye was rotated inferonasally with a bridle suture. Careful blunt dissection was used to take free conjunctiva and separate it from Tenon's capsule. A 50\% partial thickness, rectangular scleral flap was created with a sclerotome. Mitomycin C (MMC) 
$(0.2 \mathrm{mg} / \mathrm{mL})$ was placed onto the sclera with a Weck-Cel sponge (Beaver Visitec, Waltham, Massachussetts) for 5 minutes followed by copious irrigation. After a paracentesis, a clear corneal incision was made underneath the scleral flap with a $15^{\circ}$ slit blade. A Kelley punch was used to create the trabeculectomy. A surgical iridectomy was performed. The scleral flap was then sutured into place usually with two $10-0$ nylon sutures at the corners of the flap, allowing for a small flow of aqueous through the posterior edge of the flap. Careful, watertight closure of the conjunctiva was accomplished with a 9-0 vicryl suture. At the end of each case, topical neomycin/ polymyxin B/dexamethasone ophthalmic and atropine ointment were applied.

Residents were permitted to perform glaucoma surgery as primary surgeons only after the supervising attending felt confident in a resident's surgical skills, usually via performance in phacoemulsification. Because all procedures were supervised by the same attending supervisor, the surgical techniques and postoperative management employed were consistent throughout the study.

Data were recorded and analyzed using MS Excel (Microsoft, Redmond, WA). For statistical analysis, simple descriptive statistics, such as mean, range, and standard deviation were calculated for all outcome variables. Snellen BCVA was converted to logMAR for statistical analysis. For comparisons of categorical variables, the chisquare test or Fischer's exact test was performed, while the Student's t-test, Mann-Whitney U test, or Analysis of Variance (ANOVA) was performed for continuous variables. All analyses were two-way, and significance was defined as p-value of 0.05 or less.

\section{RESULTS}

In total, 272 glaucoma surgeries were performed by thirdyear ophthalmology residents between 2005 and 2012. A total of 103 eyes of 91 patients were included, with 44 receiving an Ahmed valve and 59 receiving a trabeculectomy (Table 1). Patients were of mean age of $68.1 \pm 12.3$ and $61.6 \pm 9.1$ years for valve and trab respectively $(p=0.005)$. In both the groups, majority of the patients were male and African American $(p \geq 0.25)$. Majority of the patients also carried a diagnosis of hypertension as documented in their medical record. Proportionally, more patients in the valve group carried a diagnosis of diabetes mellitus ( $p=0.002)$; however, the mean glycosylated hemoglobin A1c values within 6 months of surgery were not statistically significant between groups $(p=0.10)$.

Specific glaucoma diagnoses in each group are summarized in Table 2. Eyes receiving a valve more commonly carried a diagnosis of neovascular glaucoma
Table 1: Demographic characteristics of study participants

\begin{tabular}{llllr}
\hline & & Valve & Trab & p-value \\
\hline \multirow{4}{*}{$\begin{array}{llll}\text { Age, mean } \\
\text { (SD), y }\end{array}$} & & $(\mathrm{n}=44)$ & $(\mathrm{n}=59)$ & \\
Sex & Male & $68.1 \pm 12.3$ & $61.6 \pm 9.2$ & $<0.01$ \\
& Female & 43 & 56 & 0.64 \\
Race & African-American & 30 & 3 & \\
& Caucasian & 14 & 12 & 0.25 \\
& Hispanic & 0 & 0 & \\
& Other & 0 & 0 & \\
Hypertension & No. (\%) & $39(89)$ & $46(78)$ & 0.20 \\
Diabetes & No. (\%) & $29(66)$ & $22(37)$ & $<0.01$ \\
& A1c \% & 7.98 & 7.17 & 0.10 \\
\hline
\end{tabular}

Valve: Ahmed valve; Trab: Trabeculectomy

Table 2: Glaucoma diagnosis of eyes receiving valve and trab

\begin{tabular}{lllc}
\hline Diagnosis & Valve & Trab & $p$-value \\
\hline POAG & 16 & 57 & $<0.001$ \\
Neovascular & 18 & 0 & $<0.001$ \\
Uveitic glaucoma & 3 & 0 & 0.08 \\
Chronic angle closure & 1 & 0 & 0.43 \\
Other (OHT, Pseudoexfoliation) & 6 & 2 & 0.07 \\
\hline
\end{tabular}

at 41 vs $0 \%$ for trabeculectomy $(\mathrm{p}<0.001)$. For the trabeculectomy group, 97\% carried a diagnosis of primary open-angle glaucoma (POAG) vs 36\% for valves ( $<<0.001$ ). Other diagnoses, including uveitic glaucoma and chronic angle closure, were not statistically different between groups $(\mathrm{p}>0.07)$.

Preoperatively, mean IOP was higher in eyes receiving a valve than trabeculectomy at $35.1 \pm 11.8$ and $24.5 \pm 7.1 \mathrm{~mm} \mathrm{Hg}$ for valve and trabeculectomy respectively $(\mathrm{p}<0.001)$ (Table 3). Preoperative logMAR visual acuity was higher, indicating worse visual acuity, for eyes receiving a valve by $0.775(\mathrm{p}<0.001)$; however, the mean $\mathrm{C} / \mathrm{D}$ ratio was smaller in the valve group by $0.15(\mathrm{p}=0.002)$. The number of ocular antihypertensive medications did not differ statistically between groups $(\mathrm{p}=0.48)$.

At 1 year postoperatively, mean IOP for valve and trabeculectomy were $18.2 \pm 8.3$ and $15.3 \pm 4.5 \mathrm{~mm} \mathrm{Hg}$

Table 3: Preoperative and 1 year postoperative metrics for Ahmed valve and trabeculectomy

\begin{tabular}{llllc}
\hline & & Valve & Trab & p-value \\
\hline Pre-op & IOP & $35.1 \pm 11.8$ & $24.5 \pm 7.1$ & $<0.001$ \\
& No. of meds & $3.2 \pm 1.3$ & $3.4 \pm 0.9$ & 0.48 \\
& Visual acuity & $1.363 \pm 0.99$ & $0.588 \pm 0.742$ & $<0.001$ \\
& C/D ratio & $0.72 \pm 0.26$ & $0.87 \pm 0.10$ & 0.002 \\
Post-op & IOP & $18.2 \pm 8.3$ & $15.3 \pm 4.5$ & 0.064 \\
& No. of meds & $2.3 \pm 1.2$ & $2.2 \pm 1.5$ & 0.944 \\
& Visual acuity & $1.35 \pm 1.16$ & $0.57 \pm 0.69$ & 0.001 \\
& C/D ratio & $0.81 \pm 0.20$ & $0.88 \pm 0.09$ & 0.12 \\
\hline
\end{tabular}

IOP: Intraocular pressure $(\mathrm{mm} \mathrm{Hg})$. Visual acuity reported as logMAR 
Table 4: Changes in metrics from preoperative baseline to 1 year postoperatively

\begin{tabular}{llclc}
\hline & Valve & $p$-value & Trab & $p$-value \\
\hline IOP & $-16.9 \pm 14.7$ & $<0.001$ & $-9.2 \pm 7.5$ & $<0.001$ \\
No. of meds & $-1.0 \pm 1.5$ & 0.001 & $-1.2 \pm 1.6$ & $<0.001$ \\
Visual acuity & $-0.01 \pm 0.68$ & 0.34 & $-0.02 \pm 0.28$ & 0.81 \\
C/D ratio & $0.09 \pm 0.23$ & 0.19 & $0.01 \pm 0.06$ & 0.20 \\
\hline
\end{tabular}

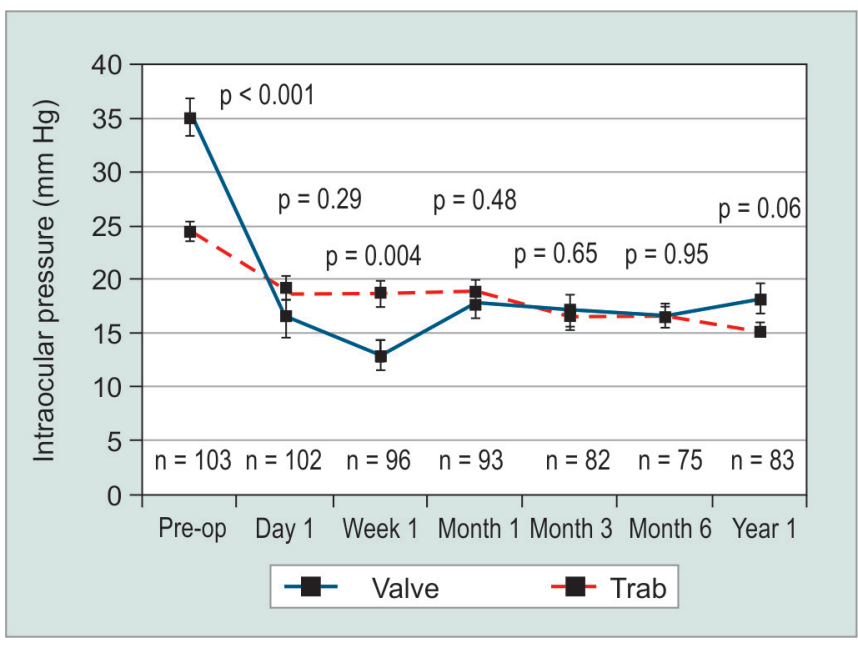

Graph 1: Mean intraocular pressure ( \pm SEM) curve over 1 year for Ahmed valve (solid line) and trabeculectomy (dashed line). $\mathrm{N}=$ number of eyes measured at a particular time point

respectively $(\mathrm{p}=0.064)$ (Table 3$)$. Neither the mean number of ocular antihypertensive medications or $\mathrm{C} / \mathrm{D}$ ratio was statistically different between groups at 1 year $(p>0.12)$. However, the mean logMAR visual acuity remained significantly poorer in the valve group, measuring $1.35 \pm 1.16$ and $0.57 \pm 0.69$ for valve and trabeculectomy respectively $(\mathrm{p}=0.001)$. When comparing preoperative to 1 year postoperative IOP, both surgeries demonstrated statistically significant reductions in IOP, although the magnitude was greater for valve than trabeculectomy at $16.9 \pm 14.7$ and $9.2 \pm 7.5 \mathrm{~mm} \mathrm{Hg}$ respectively $(\mathrm{p}<0.001)$ (Table 4). The IOP curve over 1 year for each group is illustrated in Graph 1.

Regarding rates of treatment failure, $22.5 \%$ of eyes with valve had IOP $>21 \mathrm{~mm} \mathrm{Hg}$ on two occasions after 3 months postoperatively vs only $4.3 \%$ of eyes with trabeculectomy $(\mathrm{p}=0.02)$ (Table 5). Alternatively, $15.0 \mathrm{vs}$ $21.3 \%$ exhibited $<20 \%$ IOP reduction for valve and trabeculectomy respectively, although the difference did not reach statistical significance $(p=0.28)$. Neither group had eyes with IOP $<5 \mathrm{~mm} \mathrm{Hg}$ on two occasions after 3 months postoperatively. Overall, $11(25.0 \%)$ valves and $11(18.6 \%)$ trabeculectomies met any of the three criteria for failure.

Complications from either type of surgery were overall infrequent. However, complication profiles differed for each type of surgery, as shown in Table 6. There were no intraoperative complications for valves. Four trabeculectomies were complicated by formation of buttonholes upon conjunctiva closure. The most common immediate complication for a valve implantation was hyphema (55\%); while for trabeculectomy, overfiltration $(33 \%)$ and hyphema (33\%) predominated. Within 1 week, one eye receiving trabeculectomy developed a choroidal effusion $v s$ none receiving valve. However, from 1 week to 3 months, five eyes receiving valve developed a choroidal effusion vs only one receiving trabeculectomy. Over 1 year, five eyes that received trabeculectomy developed a cataract $v s$ only one that received valve.

Regarding the need to return to the operating room, three eyes that received a valve underwent reoperation within the first 3 months $v s$ none who underwent trabeculectomy. After 3 months, one eye with a valve and two with trabeculectomy required operative intervention.

\section{DISCUSSION}

In this study, Ahmed valve implantation and trabeculectomy were shown to be both safe and effective when performed by residents-in-training under the supervision of a glaucoma specialist at a Veterans Affairs Medical Center. While multiple other studies have compared outcomes of Ahmed valves and trabeculectomy, ${ }^{9,16,17}$ this is the first study comparing not only IOP changes but also complication rates of these two glaucoma surgeries when performed by residents.

Both surgical groups in this study had significant reductions in IOP over 1 year. The magnitude of reduction was greater for valves, but this was likely due to the higher mean preoperative IOP in valve group. By 12 months, there was statistical difference in IOP between valve and trabeculectomy groups. In a prospective study of 117 patients comparing Ahmed valve with trabeculectomy when performed by glaucoma specialists, trabeculectomy maintained lower IOP than valves, with

Table 5: Rates of treatment failure for valve and trabeculectomy for patients evaluated on two occasions after 3 months postoperatively

\begin{tabular}{|c|c|c|c|c|c|c|}
\hline & \multicolumn{3}{|c|}{ Valve } & \multicolumn{3}{|c|}{ Trab } \\
\hline & $1 O P>21 \mathrm{~mm} \mathrm{Hg}$ & $<20 \% 10 P$ reduction & $I O P<5 \mathrm{~mm} \mathrm{Hg}$ & $I O P>21 \mathrm{~mm} \mathrm{Hg}$ & $<20 \%$ IOP reduction & $I O P<5 \mathrm{~mm} \mathrm{Hg}$ \\
\hline No. of eyes $(n)$ & 9 & 6 & 0 & 2 & 10 & 0 \\
\hline Total included (n) & 40 & 40 & 40 & 47 & 47 & 47 \\
\hline No. excluded (n) & 4 & 4 & 4 & 12 & 12 & 12 \\
\hline Percent failure (\%) & 22.5 & 15.0 & 0 & 4.3 & 21.3 & 0 \\
\hline
\end{tabular}


Table 6: Postoperative complications for each group over 1 year recorded as $n(\%)$. Percentages relate to total number of complications for a particular surgery in the given timeframe

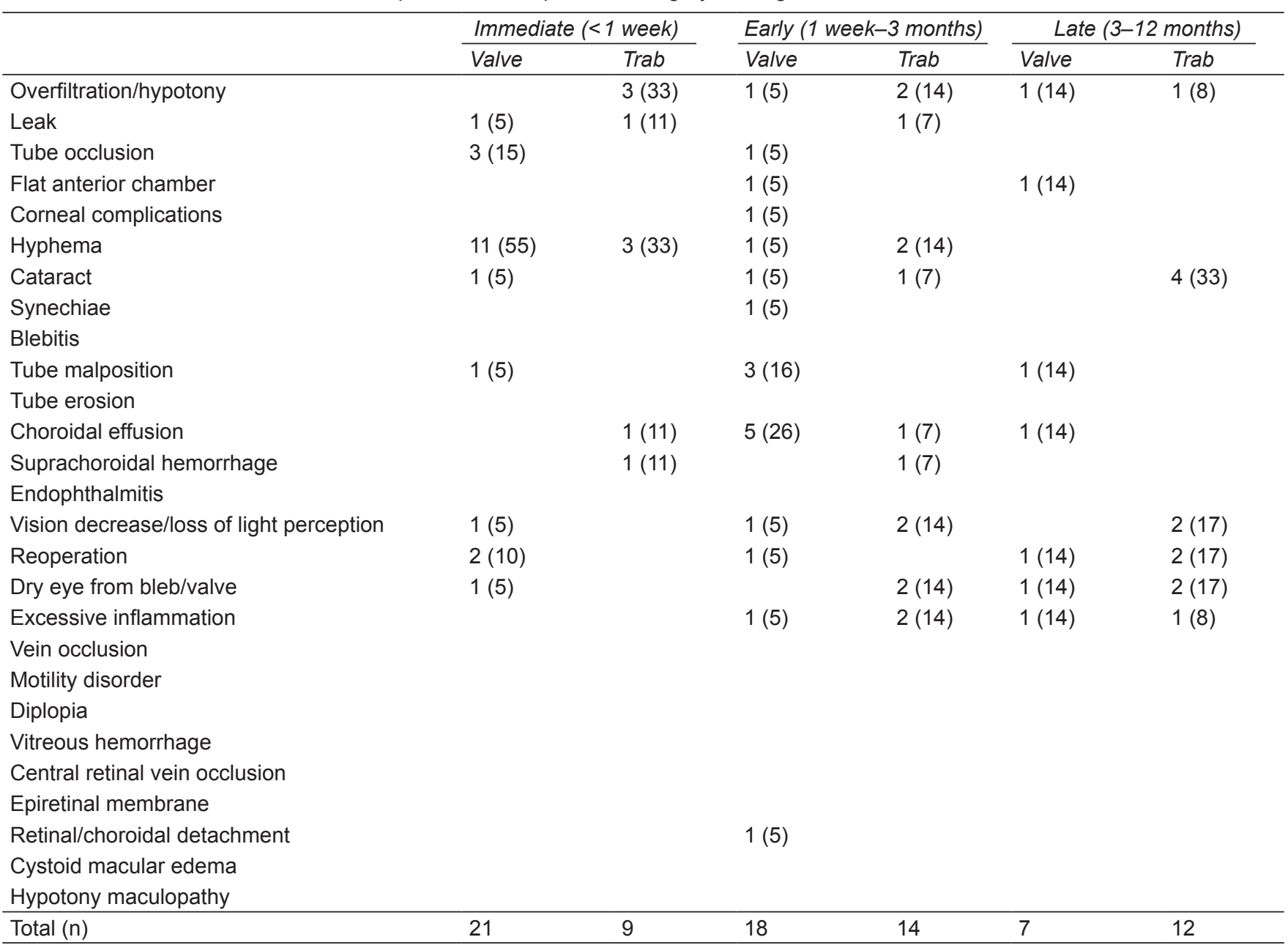

the difference being statistically significant by 1 year. ${ }^{9}$ However, when that same cohort was evaluated in a longterm follow-up study, there was no difference found in IOP between groups by months 41 to $52 .{ }^{16}$ An important difference between their cohort and the present study is the baseline characteristics of the participants, who were on average approximately 10 years younger and more evenly divided by sex. Glaucoma diagnosis was also more matched between surgical groups, and the trab group had lower mean preoperative IOP, making these eyes arguably more favorable in terms of prognosis.

Based on criteria used in the TVT trial for treatment failure, ${ }^{15}$ the valve group had more failures due to persistent IOP > $21 \mathrm{~mm} \mathrm{Hg}$ than trabeculectomy, while more in the trab group producing a less than $20 \%$ reduction in IOP. Overall failure rates were higher in valve group. Again, this is likely a result of higher mean IOP in the valve group and also more severe disease processes on average in that group. The fewer eyes with IOP reduction by at least $20 \%$ in the trabeculectomy group may be due to the lower preoperative IOP in that group. No eyes were classified as treatment failures based on having
IOP $<5 \mathrm{~mm} \mathrm{Hg}$ on two consecutive visits. In the TVT study, Gedde et $\mathrm{al}^{15}$ reported 4 and $13 \%$ rates for treatment failure for tube vs trabeculectomy respectively. Not only did the TVT include Baerveldt tube shunt instead of Ahmed valve, but also the vast majority of patients had POAG in both the groups. The difference in treatment failure for tube shunts compared with TVT may have to do with different disease process in the valve group.

Connor et $\mathrm{al}^{14}$ carried out a similar investigation comparing the outcomes of trainee-performed glaucoma surgery. However, similar to the TVT study, trabeculectomy was compared with the nonvalved Baerveldt. A total of 153 patients were included and followed retrospectively over 1 year, and similar to this study, the authors found that although the preoperative IOP was higher in the tube shunt group, postoperative IOP was similar between groups.

The type and rate of surgery complications were a primary focus of this study. There were no intraoperative complications in the valve group, while four trabeculectomies were complicated by formation of buttonholes 
during conjunctiva closure. This difference is most likely secondary to the surgical technique. With valve surgery, the conjunctiva and Tenon's capsule are removed as a unit to make a thicker covering for the valve. In a trabeculectomy, the conjunctival dissection off Tenon's capsule to create a thin bleb is a more delicate technique, which may make buttonhole formation more likely. All of the buttonholes were repaired primarily and none of those eyes experienced leaks postoperatively. In another eye, the corner of the trabeculectomy flap was accidentally truncated; however, the surgery was completed successfully and the eye had no postoperative complications and an IOP of $9 \mathrm{~mm} \mathrm{Hg}$ at 12 months.

Postoperative complications were stratified into three groups based on postoperative timeframe: Immediate $(<1$ week), early (1 week to 3 months), and late complications (3-12 months). Eyes receiving an Ahmed valve seemed to have more immediate postoperative complications than trabeculectomy, mostly due to hyphema. In the valve group, hyphema occurred in a total of $12(27.3 \%)$ patients and accounted for $55 \%$ of immediate postoperative complications. Prior studies have demonstrated hyphema occurring in 3 to $9 \%$ of valve implantation when performed by glaucoma specialists. ${ }^{10,18,19}$ One plausible explanation for the higher rate of hyphema is that more of the eyes receiving valve had neovascular glaucoma and therefore more new, friable vessels that were easily disrupted during surgery. Five eyes $(8.5 \%)$ in the trabeculectomy group had hyphema, which corresponds to published rates of 13 to $16 \%{ }^{9,20}$

Overfiltration can result in hypotony, defined as IOP less than episcleral venous pressure, ${ }^{21}$ which can result in serious complications, namely, choroidal effusions. For clinical purposes, eyes with IOP $<5 \mathrm{~mm} \mathrm{Hg}$ are typically considered hypotonous, and overall, in this study, rates were low. Hypotony was more common in the trabeculectomy group, occurring in $6(10.2 \%)$ eyes vs 2 (4.5\%) in the valve group. Cases occurred equally early and late in trabeculectomy but only occurred after 1 week in the valve group. One eye with valve did develop choroidal detachment in setting of hypotony. The other hypotonous eye in the valve group had severe neovascular glaucoma, preoperative visual acuity of count fingers, and was pre-phthisic by 1 year. All the cases in the trabeculectomy group were transient and improved by the next visit, except for two, which developed peripheral choroidal effusions. In prior studies, hypotony complicated approximately 32 to $45 \%$ of trabeculectomies by glaucoma specialists. ${ }^{9,20}$ When performed by residents, hypotony has been reported at a rate of 18 to $20 \%$ with clinical significance ranging from being a transient problem to requiring intervention and also returning to operating room for drainage of choroidals. ${ }^{14,22}$ This study is consistent in showing that residents tend to have lower rates of hypotony.

Wound leaks were rare in this study with only two instances in the trabeculectomy group and one in the valve group. The one in the valve group was only slightly Seidel positive and resolved with conservative measures. For trabeculectomy, one leak was treated with suture placement at the slit lamp. The other leak occurred in week 1 in an eye that was hypotonous immediately postoperative but was Seidel negative at that time. Studies have reported rate of wound leak at 9.7 and $0.7 \%$ for $\operatorname{trab}^{9,20}$ and valve ${ }^{18}$ respectively. No eyes developed endophthalmitis during the follow-up period.

Cataract development as a complication from surgery was uncommon but occurred more frequently in the trabeculectomy group: In five eyes (8.5\%) vs two $(4.5 \%)$ in the trabeculectomy and valve groups respectively. One eye in the valve group developed a cataract within the first week postoperatively and subsequently underwent cataract extraction. Overall, reoperation for any reason was necessary for $3.4 \%$ of trabeculectomy eyes and $9.1 \%$ of valves.

Following trabeculectomy, one eye developed a choroidal effusion and another developed suprachoroidal hemorrhage within a week postoperatively. Five eyes (11.4\%) receiving Ahmed valve developed choroidal effusions from 1 week to 3 months postoperatively. The higher incidence of choroidal effusion in the valve group may relate to the larger average decrease in IOP since the preoperative IOP was higher in that group. With the published incidence choroidal effusion of 13 to 16 and 3 to $7 \%$ for valve $\mathrm{e}^{10,18}$ and trab ${ }^{9,20}$ respectively when performed by glaucoma specialists, the rate of this serious complication is within acceptable range when performed by residents.

Mean visual acuity was significantly worse preoperatively for eyes in the valve group. This difference persisted at 1 year; however, the VA in the valve group did not worsen more on average than trab over 1 year. This difference is likely explained by the predominance of neovascular glaucoma in the valve group, which portends worse visual outcomes. The $\mathrm{C} / \mathrm{D}$ ratio was noted to be higher in the trab group preoperatively, which may be due to the fact that a larger portion of these patients had POAG and, therefore, likely a longer duration of disease. By 1 year, the $C / D$ ratio was not statistically different between groups, indicating an increase in cupping in the valve group, which again reflects the larger portion of patients with more severe and acute secondary glaucoma. Each group experienced a statistically significant reduction in number of ocular antihypertensive medications by 1 year.

This study has several limitations. First, it is retrospective nature. Also, interpretation of the results of this investigation as a head-to-head evaluation of the Ahmed valve $v s$ trabeculectomy is limited due to the different baseline characteristics of the eyes studied. With a larger 
portion of patients with diabetes and secondary glaucoma, specifically neovascular, in eyes receiving valve, the postoperative course may be altered based on underlying pathologic differences. Logically, one would expect more complications and possibly worse outcomes, which was not the case in this study. In addition, the demographic characteristics of the study group may be unique to the veteran population in this region and, therefore, may not be generalizable to other patient groups.

To date, this is the first known investigation of outcomes following Ahmed valve implantation vs trabeculectomy by residents-in-training. The results are encouraging that resident surgeons can safely perform these challenging surgeries and produce a satisfactory result with a good safety profile. Despite an influx of recent publications on resident-performed glaucoma procedures, ${ }^{14,22-27}$ a significant gap in the literature remains evaluating the plethora of procedures that residents perform on patients during their training. Not only will this research help monitor and shape surgical education, but it can also serve to provide evidence-based counseling for patients that supervised, resident-performed procedures are indeed safe and effective.

\section{CONCLUSION}

Ahmed valve implantation and trabeculectomy produce significant reductions in IOP when performed by residentsin-training. Although complication profiles differ between procedures, both are safe and well tolerated when performed by resident physicians. This study provides support for evidence-based patient counseling that supervised, resident-performed Ahmed valve implantation and trabeculectomy are indeed safe and effective.

\section{REFERENCES}

1. Congdon N, O'Colmain B, Klaver CC, Klein R, Munoz B, Friedman DS, Kempen J, Taylor HR, Mitchell P, Eye Diseases Prevalence Research Group. Causes and prevalence of visual impairment among adults in the United States. Arch Ophthalmol 2004 Apr;122(4):477-485.

2. Boland MV, Ervin AM, Friedman DS, Jampel HD, Hawkins BS, Vollenweider D, Chelladurai Y, Ward D, Suarez-Cuervo C, Robinson KA. Comparative effectiveness of treatments for open-angle glaucoma: a systematic review for the U.S. Preventive Services Task Force. Ann Intern Med 2013 Feb 19;158(4):271-279.

3. Patel S, Pasquale LR. Glaucoma drainage devices: a review of the past, present, and future. Semin Ophthalmol 2010 Sep-Nov;25(5-6):265-270.

4. Quigley HA, Cassard SD, Gower EW, Ramulu PY, Jampel HD, Friedman DS. The cost of glaucoma care provided to Medicare beneficiaries from 2002 to 2009. Ophthalmology 2013 Nov; 120(11):2249-2257.

5. Cairns JE. Trabeculectomy. Preliminary report of a new method. Am J Ophthalmol 1968 Oct;66(4):673-679.
6. Joshi AB, Parrish RK II, Feuer WF. 2002 survey of the American Glaucoma Society: practice preferences for glaucoma surgery and antifibrotic use. J Glaucoma 2005 Apr;14(2):172-174.

7. Topouzis F, Coleman AL, Choplin N, Bethlem MM, Hill R, Yu F, Panek WC, Wilson MR. Follow-up of the original cohort with the Ahmed glaucoma valve implant. Am J Ophthalmol 1999 Aug;128(2):198-204.

8. Coleman AL,HillR, Wilson MR, ChoplinN,Kotas-NeumannR, Tam M, Bacharach J, Panek WC. Initial clinical experience with the Ahmed Glaucoma Valve implant. Am J Ophthalmol 1995 Jul;120(1):23-31.

9. Wilson MR, Mendis U, Smith SD, Paliwal A. Ahmed glaucoma valve implant $v$ s trabeculectomy in the surgical treatment of glaucoma: a randomized clinical trial. Am J Ophthalmol 2000 Sep;130(3):267-273.

10. Christakis PG, Kalenak JW, Zurakowski D, Tsai JC, Kammer JA, Harasymowycz PJ, Ahmed II. The Ahmed Versus Baerveldt study: one-year treatment outcomes. Ophthalmology 2011 Nov;118(11):2180-2189.

11. ACGME. Required minimum number of procedures for graduating residents in ophthalmology [ACGME Website]. 2013. Availablefrom: http://www.acgme.org/acgmeweb/Portals/0/ PFAssets/ProgramResources/240_Oph_Minimum Numbers.pdf.

12. Golden RP, Krishna R, DeBry PW. Resident glaucoma surgical training in United States residency programs. J Glaucoma 2005 Jun;14(3):219-223.

13. Johnstone MA, Shingleton BJ, Crandall AS, Brown RH, Robin AL. Glaucoma surgery treatment patterns of ASCRS members - 2000 survey. J Cataract Refract Surg 2001 Nov;27(11):1864-1871.

14. Connor MA, Knape RM, Oltmanns MH, Smith MF. Trainee glaucoma surgery: experience with trabeculectomy and glaucoma drainage devices. Ophthalmic Surg Lasers Imaging 2010 Sep-Oct; 41(5):523-231.

15. Gedde SJ, Schiffman JC, Feuer WJ, Herndon LW, Brandt JD, Budenz DL. Treatment outcomes in the tube versus trabeculectomy study after 1 year of follow-up. Am J Ophthalmol 2007 Jan;143(1):9-22.

16. Wilson MR, Mendis U, Paliwal A, Haynatzka V. Long-term follow-up of primary glaucoma surgery with Ahmed glaucoma valve implant versus trabeculectomy. Am J Ophthalmol 2003 Sep;136(3):464-470.

17. Tran DH, Souza C, Ang MJ, Loman J, Law SK, Coleman AL, Caprioli J. Comparison of long-term surgical success of Ahmed Valve implant versus trabeculectomy in open-angle glaucoma. Br J Ophthalmol 2009 Nov;93(11):1504-1509.

18. Budenz DL, Barton K, Feuer WJ, Schiffman J, Costa VP, Godfrey DG, Buys YM, Ahmed Baerveldt Comparison Study Group. Treatment outcomes in the Ahmed Baerveldt Comparison Study after 1 year of follow-up. Ophthalmology 2011 Mar;118(3):443-452.

19. Barton K, Gedde SJ, Budenz DL, Feuer WJ, Schiffman J, Ahmed Baerveldt Comparison Study Group. The Ahmed Baerveldt Comparison Study methodology, baseline patient characteristics, and intraoperative complications. Ophthalmology 2011 Mar;118(3):435-442.

20. Wagschal LD, Trope GE, Jinapriya D, Jin YP, Buys YM. Prospective randomized study comparing Ex-PRESS to trabeculectomy: 1-year results. J Glaucoma 2015 Oct-Nov;24(8):624-629.

21. Pederson JE. Ocular hypotony. Trans Ophthalmol Soc UK 1986;105(Pt 2):220-226. 
22. Chan CK, Lee S, Sangani P, Lin LW, Lin MS, Lin SC. Primary trabeculectomy surgery performed by residents at a county hospital. J Glaucoma 2007 Jan;16(1):52-56.

23. Kwong A, Law SK, Kule RR, Nouri-Mahdavi K, Coleman AL, Caprioli J, Giaconi JA. Long-term outcomes of residentversus attending-performed primary trabeculectomy with mitomycin C in a United States residency program. Am J Ophthalmol 2014 Jun;157(6):1190-1201.

24. Seider MI, Rofagha S, Lin SC, Stamper RL. Residentperformed Ex-PRESS shunt implantation versus trabeculectomy. J Glaucoma 2012 Sep;21(7):469-474.
25. Lowry EA, Greninger DA, Porco TC, Naseri A, Stamper RL, Han Y. A comparison of Resident-Performed Argon and Selective Laser Trabeculoplasty in patients with open-angle glaucoma. J Glaucoma 2016 Mar;25(3):e157-e161.

26. Greninger DA, Lowry EA, Porco TC, Naseri A, Stamper RL, Han Y. Resident-performed selective laser trabeculoplasty in patients with open-angle glaucoma. JAMA Ophthalmol 2014 Apr 1;132(4):403-408.

27. Desai RU, Pekmezci M, Tam D, Song J, Lin SC. Residentperformed Ahmed glaucoma valve surgery. Ophthalmic Surg Lasers Imaging 2010 Mar-Apr;41(2):222-227. 